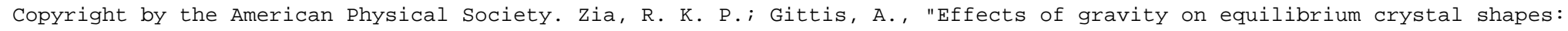
Droplets hung on a wall," Phys. Rev. B 35, 5907 (R) DOI: http://dx.doi.org/10.1103/PhysRevB.35.5907

\title{
Effects of gravity on equilibrium crystal shapes: Droplets hung on a wall
}

\author{
R. K. P. Zia and A. Gittis \\ Center for Transport Theory and Mathematical Physics and Physics Department, \\ Virginia Polytechnic Institute and State University, Blacksburg, Virginia 24061
}

(Received 12 December 1986)

\begin{abstract}
General properties of equilibrium crystal shapes pinned on a vertical wall and subject to gravity are sought. For two-dimensional crystals, or three-dimensional ones with axial symmetry held in suitable geometries, we are able to express the results in terms of the well-known gravity-free Wulff-Winterbottom shapes. All results are valid for an arbitrary, given, orientation-dependent surface-tension function.
\end{abstract}

The century-old subject of equilibrium crystal shapes ${ }^{1,2}$ has received considerable recent interest, both experimentally ${ }^{3}$ and theoretically. ${ }^{4}$ On the experimental side, technologies far superior to those of Wulff allow the observation of crystals in equilibrium with their surrounding medium. Theoretically, their relationship to surface roughening and other subtle phase transitions in two dimensions ${ }^{5}$ entices researchers from many fields.

To start from a microscopic Hamiltonian and end at an equilibrium crystal shape is, at present, a two-step process: (a) Derive $\sigma(\mathbf{n})$, the free energy per unit area associated with a flat interface with normal $\mathbf{n}$ between the crystal and the medium and (b) put $\sigma$ into the Wulff construction to come up with the equilibrium crystal shape.

While we do not have a rigorous justification for this two-step procedure, it is widely believed to be correct for macroscopic crystals whose smallest radius of curvature is much larger than the lattice constant.

Two difficulties still remain when we wish to compare theoretical predictions to earth-bound experiments. One is inherent in step (a), although many models (such as solid on solid and mean field) are deemed adequate for computing $\sigma$. The other is the effect of external forces, such as gravity, which spoils step (b), even if an exact $\sigma$ is available. The deviation from the Wulff construction becomes more severe as the size of the crystal becomes larger. While the first difficulty is no less a problem than solving the three-dimensional Ising model, the second is no more a problem than one in the calculus of variations. In this paper, we communicate some new results in a continuing ${ }^{6}$ pursuit for solutions to the second problem.

Part of the difficulty encountered with effects of gravity is the strong dependence of solutions on the details of the supports. This is a common experience, especially for isotropic $\sigma$ cases: Water droplets on tabletops are shaped differently from those hanging from faucets. In both two and three dimensions, a large variety of situations have been explored ${ }^{7}$ for isotropic $\sigma$.

For general $\sigma$ 's and nonspherical equilibrium shapes only a few workers address the problem of gravity. Cabrera and Garcia $^{8}$ evaded the issue essentially. Avron, Taylor, and $\mathrm{Zia}^{6}$ considered sessile drops, i.e., crystals supported by a homogeneous flat horizontal surface such as a tabletop. For an arbitrary given $\sigma$, exact solutions (quad- rature) are found in two dimensions while general properties are proved in all higher dimensions. The effect of gravitational-induced faceting was discovered and extended by Taylor ${ }^{9}$ to gravitational-induced curvature. Another group ${ }^{10}$ was more ambitious and studied crystals supported by curved supports, but with little concrete results.

Here we consider a different form of common support: pinning to a wall (vertical flat substrate). Physically, such "pinnings" frequently occur as a result of impurities which have a lower or higher interaction with the crystal than with the medium. To keep the problem simple and tractable, we consider only two dimensions with pinning at the top point of the crystal (the hung crystal). For threedimensional crystals, the cases considered here are applicable to crystals with axial symmetry, held in suitable geometries. In this Communication, we briefly indicate our methods, report the general results [which are valid for an arbitrary, given function $\sigma(\mathbf{n})$ ], and discuss their physical significance. Detailed analysis and solutions to several related problems will be presented in a later publication. ${ }^{11}$

To describe the shape of a crystal with fixed volume $V$, which is in equilibrium with its medium, we start with a given $\sigma(\mathbf{n})$, the free surface energy for the interface between the crystal and the medium. For problems in two dimensions, $\mathbf{n}$ may be parametrized by the angle it makes with the $x$ axis $\phi$. With neither gravity nor substrate, the equilibrium shape is found by Wulff.' Denote the $x$ and $y$ coordinates of the Wulff shape by $x_{W}$ and $y_{W}$. For $\sigma(\phi)$ leading to smooth shapes, these are explicitly given by ${ }^{12}$

$$
x_{W}(\phi)=\cos (\phi) \sigma-\sin (\phi)(d \sigma / d \phi)
$$

and

$$
y_{W}(\phi)=\sin (\phi) \sigma+\cos (\phi)(d \sigma / d \phi) .
$$

Otherwise, they are still uniquely determined by $\sigma$ and we will assume $\left(x_{W}, y_{W}\right)$ to be "given" in the same sense that $\sigma$ is given. Note that the units of $x_{W}$ and $y_{W}$ are those of $\sigma$ while the physical coordinates $(x, y)$ of the crystal surface are $\left(x_{W}, y_{W}\right)$ scaled to fit $V$ by a dimensionful parameter.

For a crystal shape in the absence of gravity but attached to a flat homogeneous substrate with an orientation fixed relative to the crystalline axes, we need Win- 
terbottom's ${ }^{2}$ construction. First, let the crystal and medium lie in the $x>0$ half space with the origin set at one of the two ends of the crystal. Next, let $\sigma_{\Delta}$ be the difference between the medium-substrate surface energy and the crystal-substrate one. Assume $x_{W}(\pi)<\sigma_{\Delta}<x_{W}(0)$; otherwise we would have the singular cases of complete drying or wetting. Now, the Winterbottom physical shape may be expressed in terms of Wulff's: $x=\left(x_{W}-\sigma_{\Delta}\right) / \lambda$; $y=\left[y_{W}-y_{W}\left(\phi_{0}\right)\right] / \lambda$, where $\lambda$ is a scale parameter for fitting $V$ and $\phi_{0}$ is the contact angle at the origin, satisfying $x_{W}\left(\phi_{0}\right)=\sigma_{\Delta}$. [See Fig. 1(a) for a $\sigma_{\Delta}>0$ case.] The other contact angle is denoted by $\phi_{f}$. These results come from minimizing an energy functional of, say, $x(y)$, consisting of surface energy terms [ $\int \sigma d a$ and $\int \sigma_{\Delta} \Theta(x) d y$, where $\Theta=1,0$ if $x>0, \leq 0$ ] and a Lagrangian multiplier term for the volume constraint $\left(\lambda \int x d y\right)$.

To describe a crystal pinned on a wall under the influence of gravity, we need additional terms in the energy functional corresponding to the pinning and gravity. The latter is simply $g \int y x d y$, where $g$ is the product of the acceleration due to gravity and the difference of crystal and medium densities. The former is another $\sigma$-like term,
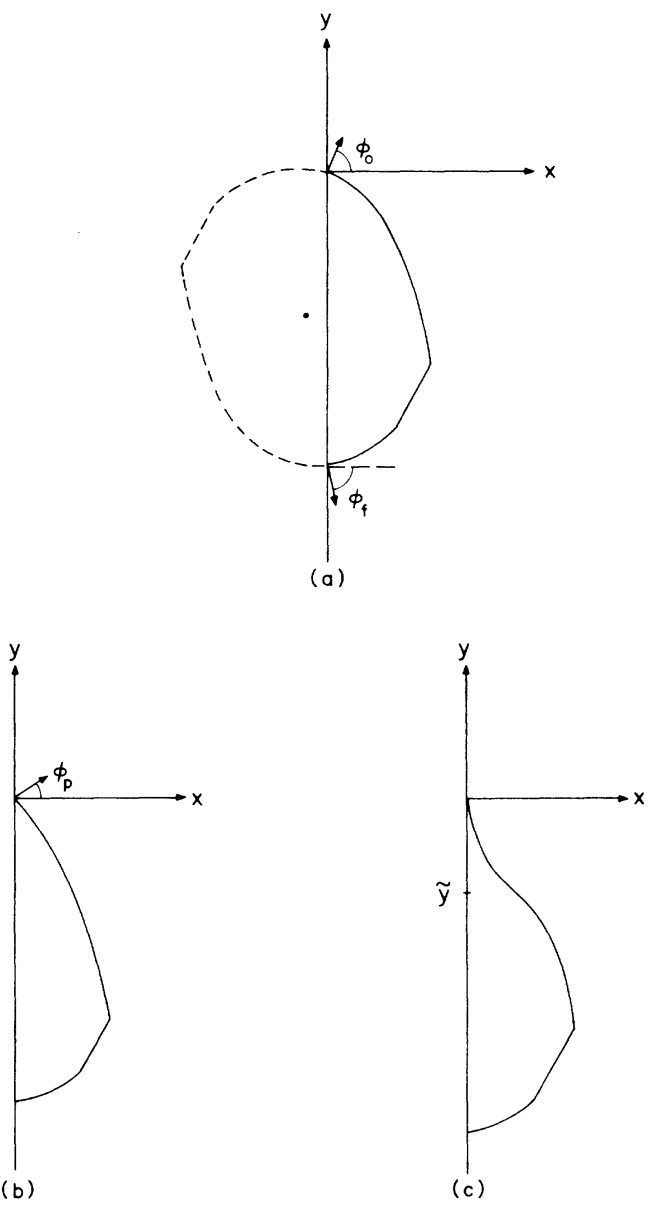

FIG. 1. (a) Winterbottom's construction for a crystal adsorbed on a substrate located at the $y$ axis, (b) a crystal hung by an impurity located at $y=0$ on an otherwise homogeneous vertical wall, (c) a hung crystal displaying a concave portion. coming from impurities on an otherwise homogeneous wall. For simplicity, we consider a single impurity localized at $y=0$. The strength, denoted by $J$, can be infinitely attractive, corresponding to a crystal being hung by its upper "edge" [cf. Fig. 1(b)]. Mathematically, we add a term $J \delta(y)$ to $\sigma_{\Delta}$ in the surface energy functional. In practice, such pinning terms translate into a boundary condition: $x(0)=0$, while the exact value of $J$ never enters. The functional to be minimized is

$$
F[x]=\int d y \Theta(x)\left\{\left[\sigma \sqrt{\left(1+\dot{x}^{2}\right)}-\sigma_{\Delta}+J \delta\right]-\lambda x+g y x\right\},
$$

where $\dot{x} \equiv d x / d y$. The sign in the volume constraint term is clearly arbitrary, though it is chosen to conform to the $g=0$ limit where $\lambda>0$. Recall that $\sigma$ can be an arbitrary function of $\phi$, which in turn depends on $\dot{x}$ via $\dot{x}=-\cot \phi$. Thus, the Euler-Lagrange equations correspond to two boundary conditions ${ }^{6,11}$ and a nonlinear differential equation of arbitrary form.

Before presenting the solution, we should remark on the mathematics of the minimization and the physics of the boundary conditions. First, because of gravity, $F$ is strictly unbounded from below, even if it is constrained to fixed $V$. It can always be lowered by breaking the crystal into two pieces, one bit over the pin and another piece running off to $y=-\infty$. So the minimum we seek is a local one, corresponding to a connected crystal. (Note also that if $\lambda$ is fixed instead of $V$, the solution to the variational problem is a saddle point. The physical meaning is that of a critical nucleation droplet needed for a first-order transition to an equilibrium state with semi-infinite regions of crystal and a medium divided by a meniscus at $y=\lambda / g$. ) Next, we comment on the two "edges" of the crystal. On the free end, $x=0$ and the contact angle is just $\phi_{f}$, while $y$ is an unknown. On the pinned end, $x=y=0$ while the contact angle $\phi_{p}$ is an unknown. These unknowns will be functions of $\lambda$ and $g$, which must be solved in terms of $V$ and $g$. Physically, we should expect that fixing $V$ and increasing $g$ (or vice versa) would change the length of the crystal and the contact angle at the pinned edge.

Though generally nonlinear, the differential equation may be solved by quadrature. Defining $K\left(\phi ; \phi_{p}\right)$ $\equiv y_{W}\left(\phi_{p}\right)-y_{W}(\phi)$, the solution is best displayed parametrically, in a form most suitable for checking the gravityfree limit:

$$
\begin{aligned}
& y(\phi)=(\lambda / g)\left\{1-\left[1+2 g \lambda^{-2} K\left(\phi ; \phi_{p}\right)\right]^{1 / 2}\right\}, \\
& x(\phi)=\int_{\phi_{f}}^{\phi} d \phi\left(d x_{W} / d \phi\right)\left[\lambda^{2}+2 g K\left(\phi ; \phi_{p}\right)\right]^{-1 / 2} .
\end{aligned}
$$

To obtain these, we have imposed part of the boundary conditions, i.e., $x\left(\phi_{f}\right)=0$ and $y\left(\phi_{p}\right)=0$. The other condition leads to an equation for the contact angle: $0=x\left(\phi_{p}\right)=\int_{\phi_{f}}^{\phi_{p}} d \phi\{\cdots\}$. This is not a simple equation for finding $\phi_{p}$ as a function of $\lambda$ and $g$. If we wish to find $\phi_{p}$ in terms of $V$ and $g$, there is the added labor of folding in whatever the functional form for $\lambda(V, g)$. Thus, it is very surprising to find the simple result

$$
g V=x_{W}\left(\phi_{p}\right)-\sigma_{\Delta} .
$$

This equation provides an easy, graphic determination of 
$\phi_{p}$, just as the Winterbottom construction determines $\phi_{0}$. Once $\phi_{p}$ is found, all quantities on the right-hand sides of (2) are explicitly known. One immediate consequence of (3) is that there is maximum value of $g V$ allowed, i.e., $x_{W}(0)-\sigma_{\Delta}$. For fixed $g$, this corresponds to a critical $V_{c}$, beyond which, there is no local minimum for $F$ (even when it is constrained to have a fixed volume). We may label this situation gravitationally induced wetting (GIW). Physically, the (connected) crystal shape consists of an indefinitely long interface hugging the wall, the price of which is more than compensated for by the gain in gravitational energy associated with the bulk of the crystal.

The hung crystal displays another interesting feature. Before $g V$ reaches its critical value, its shape develops a concave portion [Fig. 1(c)]. This phenomenon occurs at a $V^{*}$ for which $\lambda\left(V^{*}, g\right)=0$. For $V>V^{*}, \lambda$ is negative, so that there is a region where the effective pressure on the crystal is positive and the interface becomes concave. Indeed, the point of inflection in the crystal shape occurs at $y=\tilde{y} \equiv \lambda / g$, which is "physical" only for negative $\lambda$. The concave portion is identical to the convex part from $\tilde{y}$ to $2 \tilde{y}$, rotated by $180^{\circ} . V^{*}$ is not as easy to determine as $V_{c}$; one must first find $\zeta$ from

$$
0=\int_{\phi_{f}}^{\zeta} d \phi\left(d x_{W} / d \phi\right) / \sqrt{K\left(\phi ; \phi_{p}\right)}
$$

then $g V^{*}=x_{W}(\zeta)-\sigma_{\Delta}$. In this connection, we offer a very convenient way to measure $\sigma_{s}$, the step-free energy associated with a facet, when the crystal displays an inflection point. For $g=0$, the equilibrium shape being scale invariant, the "length" of a facet is ${ }^{4}$ proportional to, but does not determine, $\sigma_{s}$. However, $g$ provides an absolute length scale, and $\sigma_{s}$ is given by

$$
\sigma_{s}=g\left|y_{0}-\tilde{y}\right| L,
$$

where $y_{0}$ locates the center of the facet and $L$ is its length.

Finally, we emphasize that certain normals in the gravity-free equilibrium shape are absent when $g>0$. For $V<V^{*}$, those with angles between $\phi_{0}$ and $\phi_{p}$ are absent due to (3). For $V>V^{*}$, ones with angles larger than that at the inflection point are absent. The effect is especially dramatic if there are facets with $\phi$ between $\phi_{0}$ and 0 which could disappear as $g$ or $V$ is increased.

In this Rapid Communication we reported several mathematically simple and physically useful results associated with the effects of gravity on the equilibrium shape of a crystal hung on a vertical wall. We have also investigated a number of related problems: crystals supported by their lower edges, menisci pinned on a wall, inclined planar substrates, pendant drops, and crystals attached to corners of two substrates. Details of the analysis leading to the above results and studies of the related problems will be published elsewhere. ${ }^{11}$

This research is partially supported by the National Science Foundation through the Division of Materials Research.
${ }^{1}$ G. Wulff, Z. Kristallogr. Mineral. 34, 449 (1901); see also, C. Herring, in Structure and Properties of Solid Surfaces, edited by R. Gomer and C. S. Smith (Univ. of Chicago Press, Chicago, 1953).

${ }^{2}$ W. L. Winterbottom, Acta Metall. 15, 303 (1967).

${ }^{3}$ S. Balibar and B. Castaing, J. Phys. (Paris) 41, 329 (1980); J. E. Avron, L. S. Balfour, C. G. Kuper, J. Landau, S. G. Lipson, and L. S. Schulman, Phys. Rev. Lett. 45, 814 (1980); K. O. Keshishev, A. Ya Parshin, and A. V. Babkin, Zh. Eksp. Teor. Fiz. 80, 716 (1980) [Sov. Phys. JETP, 53, 362 (1982)].

${ }^{4}$ C. Rottman and M. Wortis, Phys. Rev. B 24, 6274 (1982); J. E. Avron, H. van Beijeren, L. S. Schulman, and R. K. P. Zia, J. Phys. A 15, L81 (1982); C. Jayaprakash, W. F. Saam, and S. Teitel, Phys. Rev. Lett. 50, 2017 (1983); C. Rottman and M. Wortis, Phys. Rep. 103, 59 (1984); R. K. P. Zia, in Statistical and Particle Physics-Common Problems and Techniques, edited by K. C. Bowler and A. J. McKane (Scottish Universities Summer School in Physics Publications, Edinburgh, 1984), pp. 247-301.

${ }^{5}$ R. L. Dobrushin, Theory Probab. Its Appl. (Eng. Trans.) 17, 619 (1972); J. Weeks, G. H. Gilmer, and H. J. Leamy, Phys. Rev. Lett. 31, 549 (1973); J. M. Kosterlitz and D. Thouless, J.
Phys. C 6, 1181 (1973); H. van Beijeren, Phys. Rev. Lett. 38, 993 (1977); D. R. Nelson, Phys. Rev. B 18, 2318 (1978); J. V. Jose, L. P. Kadanoff, S. Kirkpatrick, and D. R. Nelson, ibid. 16, 1217 (1978); B. I. Halperin and D. R. Nelson, Phys. Rev. Lett. 41, 121 (1978); 41, 519 (1978); Phys. Rev. B 19, 2457 (1979); H. J. F. Knops, ibid. 20, 4670 (1979).

${ }^{6}$ J. E. Avron, J. E. Taylor, and R. K. P. Zia, J. Stat. Phys. 33, 493 (1983).

${ }^{7}$ E. Pitts, J. Fluid Mech. 63, 487 (1972); D. H. Michael and P. G. Williams, Proc. R. Soc. London, Ser. A 351, 117 (1976); R. Finn, Pacific J. Math. 88, 549 (1980), and references therein.

${ }^{8}$ N. Cabrera and N. Garcia, Phys. Rev. B 25, 6057 (1982).

${ }^{9}$ J. E. Taylor, Asterisque 118, 243 (1984).

${ }^{10}$ L. A. Bol'shov, V. L. Pokrovshy, and G. V. Uimin, J. Stat. Phys. 38, 191 (1985).

${ }^{11} \mathrm{~A}$. Gittis and R. K. P. Zia (unpublished).

${ }^{12}$ W. K. Burton, N. Cabrera, and F. C. Frank, Philos. Trans. R. Soc. 243, 299 (1951); D. W. Hoffman and J. W. Chan, Surf. Sci. 31, 369 (1972); J. W. Cahn and D. W. Hoffman, Acta Metall. 22, 1205 (1974); R. K. P. Zia and J. E. Avron, Phys. Rev. B 25, 2042 (1982). 